\title{
Article
}

\section{Discrepancies in Knee Joint Moments Using Common Anatomical Frames Defined by Different Palpable Landmarks}

Thewlis, Dominic, Richards, Jim and Bower, Judith

Available at http://clok.uclan.ac.uk/3273/

Thewlis, Dominic, Richards, Jim ORCID: 0000-0002-4004-3115 and Bower, Judith (2008) Discrepancies in Knee Joint Moments Using Common Anatomical Frames Defined by Different Palpable Landmarks. Journal of Applied Biomechanics, 24 . pp. 185-190. ISSN 1065-8483

It is advisable to refer to the publisher's version if you intend to cite from the work.

For more information about UCLan's research in this area go to http://www.uclan.ac.uk/researchgroups/ and search for <name of research Group>.

For information about Research generally at UCLan please go to http://www.uclan.ac.uk/research/

All outputs in CLoK are protected by Intellectual Property Rights law, including Copyright law. Copyright, IPR and Moral Rights for the works on this site are retained by the individual authors and/or other copyright owners. Terms and conditions for use of this material are defined in the policies page.

\section{CLoK}

Central Lancashire online Knowledge www.clok.uclan.ac.uk

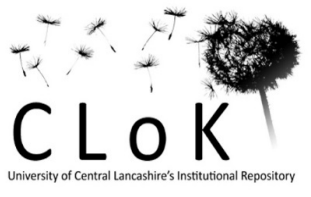




\title{
Discrepancies in Knee Joint Moments Using Common Anatomical Frames Defined by Different Palpable Landmarks
}

\author{
Dominic Thewlis, Jim Richards, and Judith Bower \\ University of Central Lancashire
}

\begin{abstract}
The aim was to investigate the effects of three anatomical frames using palpable anatomical landmarks of the knee on the net knee joint moments. The femoral epicondyles, femoral condyles, and tibial ridges were used to define the different anatomical frames and the segment end points of the distal femur and proximal tibia, which represent the origin of the tibial coordinate system. Gait data were then collected using the calibrated anatomical system technique (CAST), and the external net knee joint moments in the sagittal, coronal, and transverse planes were calculated based upon the three anatomical frames. Peak knee moments were found to be significantly different in the sagittal plane by approximately $25 \%$ ( $p \leq 0.05$ ), but no significant differences were seen in the coronal or transverse planes. Based on these findings it is important to consider the definition of anatomical frames and be aware that the use of numerous anatomical landmarks around the knee can have significant effects on knee joint moments.
\end{abstract}

Keywords: biomechanics, modeling, kinetics

The CAMARC II group (computer-aided movement analysis in a rehabilitation context; 1995) proposed recommendations for palpable anatomical landmarks in order to define the anatomical frame of the lower limbs; these were largely based on the work conducted by Cappozzo et al. (1995). This work was designed to standardize methodology and resolve many of the historical issues associated with modeling the segments of

Thewlis and Richards are with the Department of Allied Health Professionals, University of Central Lancashire, Preston, United Kingdom, and Bower is with the Department of Technology, University of Central Lancashire, Preston, United Kingdom. the lower limbs. The issue of hip joint identification is one that has been covered in much depth, and there are still many debates around this area. However, the errors associated with knee joint location have received much less attention. There have been a number of landmarks used around the knee, and some confusion may arise over which definition is correct (Vaughan et al., 1999; Davis et al., 1991) as terminology is often confused between the femoral epicondyles and the femoral condyles.

A number of authors have documented the effect of changing the position and orientation (the pose) of the segment coordinate system on knee joint moments (Holden \& Stanhope, 1998; Manal et al., 2002; Schache et al., 2007). As well as investigating the effects of moving the segment coordinate system origin, there has also been debate on the effects of orthogonal (lab, proximal segment, or distal segment systems) and nonorthogonal coordinate systems, such as joint coordinate systems (Schache \& Baker, 2007). Referencing of moments to the joint coordinate system (Grood \& Suntay, 1983) appears to be more representative of the joint function (Schache \& Baker, 2007). However, there appears to be few discrepancies in normal gait when calculating knee joint moments with respect to the distal coordinate system and in relation to a joint coordinate system. It is interesting that Schache et al. (2007) noted that lower limb transverse plane moments were particularly sensitive to variation in the reference frame. These findings were preceded by those of Holden and Stanhope (1998) and Manal et al. (2002), who both changed the pose of the tibial coordinate system by either simulating a linear displacement or inducing up to a $15^{\circ}$ rotation through the transverse plane. It is important to note that Holden and Stanhope (1998) found that a linear displacement as small as $10 \mathrm{~mm}$ can result in a change in the clinical interpretation of the joint moment. Whereas Manal et al. 
(2002) noted that a $15^{\circ}$ rotation of the tibial coordinate system can result in large differences in sagittal and coronal plane knee moments, for what they deemed to be only a small change in pose.

The aim of this study was to quantify the effect of three anatomical frames around the knee on the relative position of the origin of the tibial coordinate system and the corresponding effect found on the net knee joint moments when modeling using the calibrated anatomical systems technique (CAST).

\section{Methods}

\section{Participants}

Ten participants were recruited, all of whom were pain and pathology free. Local ethical approval was given for this study and all of the subjects gave written consent. The subjects' age ranged from 18 to 37 with a mean 22 years, height ranged from $1.54 \mathrm{~m}$ to $1.89 \mathrm{~m}$ with a mean of $1.68 \mathrm{~m}$, weight ranged from $45 \mathrm{~kg}$ to $83 \mathrm{~kg}$ with a mean of $61 \mathrm{~kg}$.

\section{Definition of the Anatomical Frame}

In order to define the local coordinate systems (LCS) within an anatomical frame, a standing static trial was collected for each anatomical landmark (Figure 1) using the principles of CAST as defined by Cappozzo et al.
(1995). The knee was identified by the segment end points of the distal femur and proximal tibia. Three marker configurations were used to define the knee: medial and lateral femoral epicondyles, femoral condyles, and the tibial ridges. The distal tibia was defined by the medial and lateral malleoli and the knee markers. The anatomical landmarks were defined using the guidelines described by Van Sint Jan and Della Croce (2005). The LCS was calculated based on the anatomical frame defined by the distal and proximal joints and the lateral joint markers.

\section{Data Collection}

Having defined the LCS, the anatomical landmarks were then removed, leaving only the cluster on the segments. Gait data were collected using a ProReflex (Qualisys medical AB, Gothenburg, Sweden) and a Kistler force platform (9281 CA, Kistler Instrumente AG, Switzerland). The kinematic data were sampled at $100 \mathrm{~Hz}$, with the force data sampled at $200 \mathrm{~Hz}$. The data were collected with the participants walking at a comfortable natural speed. Five trials were collected for each participant.

\section{Data Processing}

The raw data were exported to Visual3D (C-Motion Inc., USA). The raw trajectory and force data were then filtered using a fourth-order, low-pass, Butterworth filter with cut-off frequencies of $6 \mathrm{~Hz}$ and $25 \mathrm{~Hz}$, respectively.
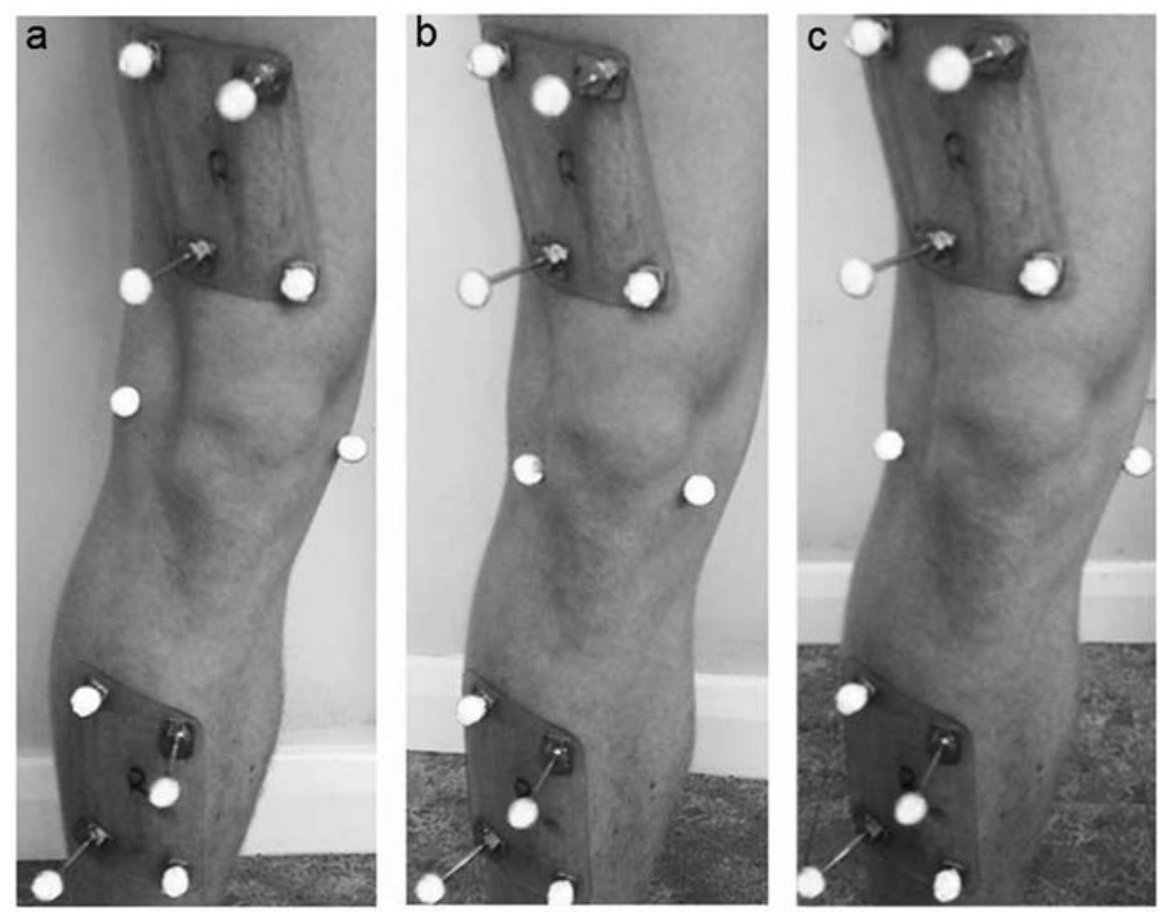

Figure 1 - Left: marker placement when using the femoral epicondyle model. Center: marker placement when using the femoral condyle model. Right: marker placement when using the tibial ridge model. 
The net external knee joint moments were then calculated for all trials using the inverse dynamic methods with respect to the tibial coordinate system, using each of the anatomical frames. This allowed for the effect of each of the landmark configurations to be used as the basis for the calculations of the net moments on the same gait trials. The moment data were normalized to body mass $(\mathrm{N} \cdot \mathrm{m} / \mathrm{kg})$ and plotted for stance phase. Knee moments were recorded at maximum flexion, maximum extension, first maximum adduction during loading, second maximum adduction during terminal stance, maximum internal torsion, and maximum external torsion moments. The ensemble means of the five trials were calculated for each of the participants. The position of the tibial coordinate system origin in relation to the foot coordinate system was calculated in the global coordinate system, providing the coordinates of the tibial coordinate system in relation to the ankle coordinate system. The global difference between the models was simply calculated as the difference between the coordinates. In order to allow for meaningful comparison and discussion of the moment data, the epicondyle model was used as a reference. It is important to note that this does not imply that the epicondyles should be deemed to be the "gold standard" for defining the knee, when there is in fact no well-defined gold standard.

Table 1 Mean (SD) Global Coordinates of the Tibial Coordinate System With Respect to the Ankle Coordinate System

\begin{tabular}{cccc}
\hline & \multicolumn{3}{c}{ Displacement $(\mathrm{m})$} \\
\cline { 2 - 4 } & $\mathbf{X}$ & $\mathbf{Y}$ & $\mathbf{Z}$ \\
\hline \multirow{3}{*}{ FE model } & 0.03 & 0.04 & 0.36 \\
& $(0.02)$ & $(0.04)$ & $(0.06)$ \\
FC model & 0.06 & 0.04 & 0.35 \\
& $(0.02)$ & $(0.03)$ & $(0.08)$ \\
TR model & 0.04 & 0.02 & 0.34 \\
& $(0.02)$ & $(0.05)$ & $(0.07)$ \\
\hline
\end{tabular}

Note. FE, femoral epicondyle; FC, femoral condyle; TR, tibial ridge.

\section{Statistical Analysis}

A repeated-measures ANOVA was performed on the maximum knee moments in the sagittal, coronal, and transverse planes and with post hoc pairwise comparison with Bonferroni adjustment. Adjusted $p$ values were reported.

\section{Results}

The average positional covariances of the knee joint center in relation to the ankle joint center, associated with the different marker systems were found to be 0.04 $\mathrm{m}, 0.03 \mathrm{~m}$, and $0.03 \mathrm{~m}$ along the $x$ (medial-lateral), $y$ (anterior-posterior), and $z$ (proximal-distal) axes relatively to the static pose of the tibia and femur (Table 1). Significant differences were noted in the sagittal plane moments at maximum flexion and maximum extension $(p<0.001)$. No further significant differences were noted in the coronal or transverse plane moments $(p>$ 0.05) (Table 2). Post hoc pairwise comparisons for the sagittal plane moments identified a significant increase between the epicondyle model and the condyle model at maximum flexion and extension $(p<0.001)$. A significant decrease was also noted at maximum extension between the epicondyle and tibial ridge models (Table 3 ). Figure 2 details the changes in knee moments associated with the different models.

\section{Discussion}

The maximum induced difference of the tibial origin with respect to that calculated using the femoral epicondyle model was found for the condyle model. The condyle model produced displacements of $30 \mathrm{~mm}$ anterior and $20 \mathrm{~mm}$ medial, and these equated to an approximately $25 \%$ difference in the flexion-extension moment, an $8 \%$ difference in the adduction-abduction moment, and a $0.5 \%$ difference in torsional moment, which suggests that transverse plane moments may not be as sensitive to changes in the reference system as previously postulated

Table 2 Mean (SD) Knee Moments and Repeated-Measures ANOVA $p$ Values

\begin{tabular}{|c|c|c|c|c|}
\hline & \multicolumn{3}{|c|}{ Mean (SD) } & \multirow{2}{*}{$\begin{array}{c}\text { Repeated-measures } \\
\text { ANOVA } \\
p\end{array}$} \\
\hline & FE model & FC model & TR model & \\
\hline Maximum flexion & $-0.70(0.37)$ & $-1.08(0.57)$ & $-0.66(0.30)$ & $<0.001$ \\
\hline Maximum extension & $0.31(0.13)$ & $0.08(0.08)$ & $0.15(0.05)$ & 0.002 \\
\hline 1 st adduction & $0.35(0.16)$ & $0.27(0.07)$ & $0.33(0.08)$ & 0.242 \\
\hline 2nd adduction & $0.31(0.17)$ & $0.30(0.12)$ & $0.30(0.10)$ & 0.940 \\
\hline $\begin{array}{l}\text { Maximum internal transverse } \\
\text { rotation }\end{array}$ & $0.15(0.05)$ & $0.14(0.05)$ & $0.15(0.05)$ & 0.533 \\
\hline
\end{tabular}

Note. Moments are reported in $\mathrm{N} \cdot \mathrm{m} / \mathrm{kg}$. FE, femoral epicondyle; FC, femoral condyle; TR, tibial ridge. 
Table 3 Post Hoc Pairwise Comparison

\begin{tabular}{|c|c|c|c|c|c|c|c|c|c|c|}
\hline \multirow[b]{2}{*}{ Comparison } & \multicolumn{2}{|c|}{ Max. flexion } & \multicolumn{2}{|c|}{ Max. extension } & \multicolumn{2}{|c|}{$\begin{array}{c}\text { 1st max. } \\
\text { adduction }\end{array}$} & \multicolumn{2}{|c|}{$\begin{array}{l}\text { 2nd max. } \\
\text { adduction }\end{array}$} & \multicolumn{2}{|c|}{$\begin{array}{c}\text { Max. internal } \\
\text { transverse rotation }\end{array}$} \\
\hline & $\begin{array}{l}\text { Mean } \\
\text { diff. }\end{array}$ & $p^{a}$ & $\begin{array}{l}\text { Mean } \\
\text { diff. }\end{array}$ & $p^{a}$ & $\begin{array}{l}\text { Mean } \\
\text { diff. }\end{array}$ & $p^{a}$ & $\begin{array}{l}\text { Mean } \\
\text { diff. }\end{array}$ & $p^{a}$ & $\begin{array}{l}\text { Mean } \\
\text { diff. }\end{array}$ & $p^{a}$ \\
\hline $\begin{array}{l}\text { Epicondyle model } \\
\text { and condyle model }\end{array}$ & $0.375^{*}$ & 0.001 & $0.233 *$ & $<0.001$ & 0.076 & 0.480 & 0.010 & 1.000 & 0.012 & 0.815 \\
\hline $\begin{array}{l}\text { Epicondyle model } \\
\text { and tibial model }\end{array}$ & -0.044 & 1.000 & $0.167 *$ & 0.012 & 0.013 & 1.000 & 0.013 & 1.000 & 0.004 & 1.000 \\
\hline $\begin{array}{l}\text { Condyle model and } \\
\text { tibial model }\end{array}$ & $-0.420 *$ & 0.007 & -0.066 & 0.228 & -0.063 & 0.250 & 0.003 & 1.000 & -0.008 & 1.000 \\
\hline
\end{tabular}

adjusted for multiple comparisons.

*The mean difference is significant at the 0.05 level.

(Schache et al., 2007). The major finding of this study was that variation in the choice of anatomical landmarks used to identify the knee joint can have a significant effect on the knee flexion-extension moments. The nature of the change of anatomical frame was different from that used by Holden and Stanhope (1998), whereby the offset was defined by anatomical landmarks and not symmetrically offset computations. The findings still support Holden and Stanhope (1998), in that a 10-mm change in the position of the coordinate system origin can have a significant effect on the knee moments. However, this positional difference can be as large as $30 \mathrm{~mm}$, and the 10-mm simulation by Holden and Stanhope (1998) is an underestimation of the potential displacement.

The knee moments in Figure 2 show larger standard deviations; this is simply due to variance between participants. Within-participant standard deviations were on average $0.03 \mathrm{~N} \cdot \mathrm{m} / \mathrm{kg}, 0.01 \mathrm{~N} \cdot \mathrm{m} / \mathrm{kg}$, and 0.015 $\mathrm{N} \cdot \mathrm{m} / \mathrm{kg}$ for the sagittal, coronal, and transverse planes, respectively. The knee flexion moment pattern showed similar moments for all reference systems at heel strike. However, as the knee flexed during loading, the femoral condyle model produced a relatively high first flexion moment, and the tibial ridge model produced a relatively low first flexion moment. The opposite effects can be seen with the first extension moment, with the moments being relatively high or low with respect to the model. This relatively low extension moment could be interpreted as a pathological gait pattern, such as quadriceps avoidance gait, when in fact the pathological pattern is simply due to marker placement. The changes in the pose of the tibial coordinate system are documented in Figure 3.

In the coronal plane, the femoral condyle model produced a discrepancy that did not match the profile of the other marker configurations. This is possibly due to the femoral condyle model producing both an anterior and lateral shift in the origin of the tibial coordinate system and segment orientation, therefore changing the static pose of the segment. This causes the segment coordinate system to be externally rotated, anteriorly and laterally translated. The lateral translation of the static tibial coordinate system origin is then represented in the low first adduction moment peak. It is important to note that although there is clearly a difference at the first peak of the adduction moment, it is not significant owing to the variability of the data. As with the results found in the sagittal plane, it is essential to note the clinical significance of the low first adduction moment and that it is due not to pathology, but marker placement.

This study further reinforces the fact that if knee joint moments are calculated, repeatable and clearly defined marker placement is essential. Although the relative distances between landmarks may be small, any incorrect identification can have effects resulting in planar cross-talk errors, resulting in larger discrepancies. The potential errors and shortcomings of current techniques suggest that new techniques be developed and implemented to optimize segment coordinate system definition. Such techniques are currently in place; however, they are underused at present. Two examples of new methods and techniques are the use of dynamic pointers, such as the dynamic pointer, and functional joint centers. Dynamic pointers allow for more accurate definition of palpable anatomical landmarks as a result of the decreased measurement errors associated with location of a point as opposed to the centroid of a marker. Functional joint center approaches (Leardini et al., 1999; Schwartz \& Rozumalski, 2005) have been around for some time. In the simplest sense, functional joint center methods calculate the center of rotation, and subsequently the origin of the segment coordinate system based on the articulation of two rigid bodies about a pivot point. Even though these techniques reduce errors associated with human factors by avoiding the necessity of locating bony landmarks, there is still limited information relating to their effectiveness for all joints of the lower limbs.

This study has highlighted a potential source of error when calculating knee joint moments; it should, however, be noted that there is no single gold standard set of anatomical landmarks. Therefore, the authors believe that the introduction of new techniques will optimize the ways in which segment coordinate systems and joint centers are identified, thus allowing for more accurate and reliable reporting of joint moments in the future. 

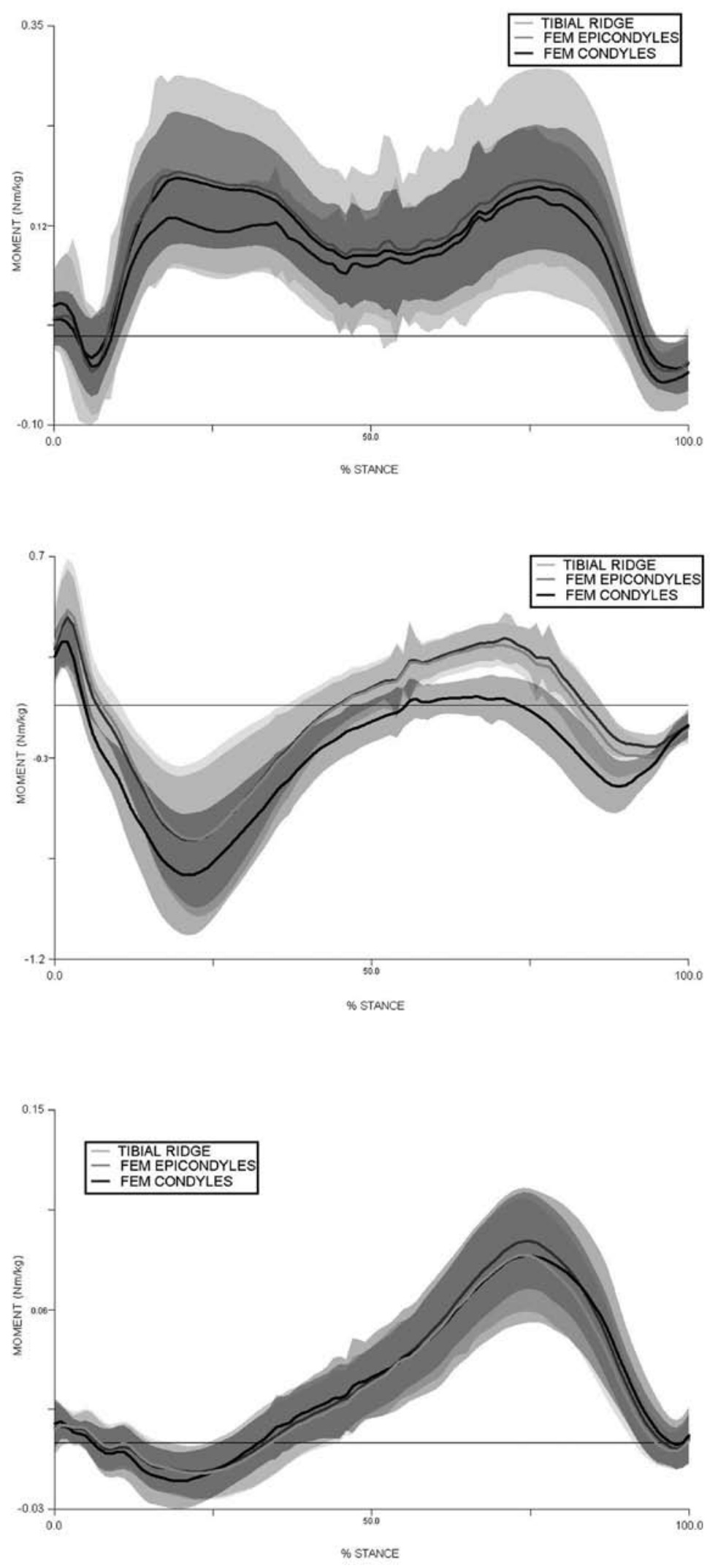

Figure 2 - Top: mean abduction-adduction moment for the three models $(+v e=$ adduction, $-\mathrm{ve}=$ abduction). Middle: mean flexion-extension moment curves for the models $(+\mathrm{ve}=$ extension, $-\mathrm{ve}=$ flexion $)$. Bottom: mean internal-external torsion moment curves for the models $(+\mathrm{ve}=$ external, $-\mathrm{ve}=$ internal $)$. 


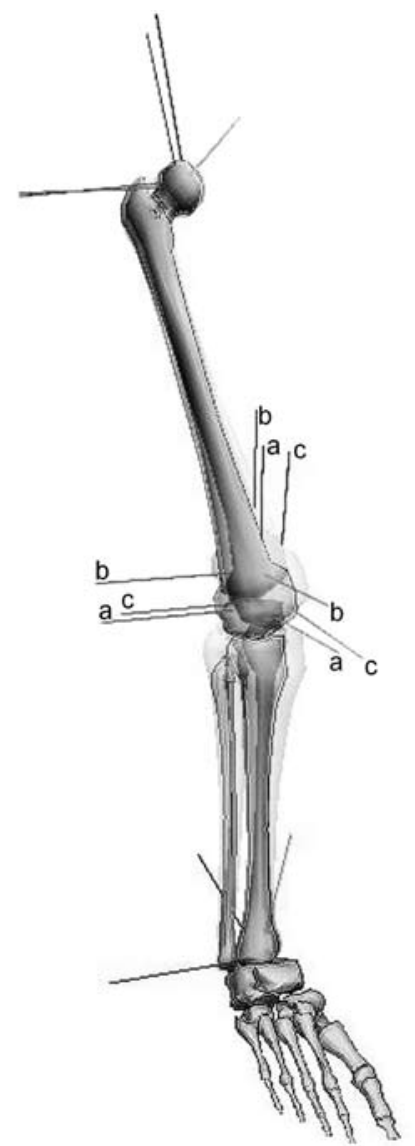

Figure 3 - Segment coordinate systems based on the different anatomical landmarks: a) femoral epicondyles, b) femoral condyles, and c) tibial ridges. Note that the difference in the segment pose is based on the different anatomical coordinate systems, the femoral condyle model results in an anterior, lateral and proximal translation, whereas the tibial ridge model results in a distal translation all with respect to the epicondyle model.

\section{References}

Cappozzo, A., Catani, F., Croce, U., \& Leardini, A. (1995). Position and orientation in space of bones during movement: anatomical frame definition and determination. Clinical Biomechanics, 10, 171-178.

Davis, R., Ounpuu, S., Tyburski, D., \& Gage, J. (1991). A gait data collection and reduction technique. Human Movement Sciences, 10, 575-587.

Grood, E., \& Suntay, W. (1983). A joint coordinate system for the clinical description of three-dimensional motions: application to the knee. Transactions of the ASME, 105, $136-144$

Holden, J., \& Stanhope, S. (1998). The effect of variation in knee centre location estimates on net knee joint moments. Gait and Posture, 7, 1-6.

Leardini, A., Cappozzo, A., Catani, F., Toksvig-Larsen, S., Petitto, A., Sforza, V., et al. (1999). Validation of a functional method for the estimation of hip joint centre location. Journal of Biomechanics, 32, 99-103.

Manal, K., McClay, I., Richards, J., Galinat, B., \& Stanhope, S. (2002). Knee moment profile during walking: errors due to soft tissue movement of the shank and the influence of the reference coordinate system. Gait and Posture, $15,10-17$.

Schache, A., Baker, R., \& Vaughan, C. (2007). Differences in lower limb transverse plane moments during gait when expressed in two alternative reference frames. Gait and Posture, 40, 9-19.

Schache, A., \& Baker, R. (2007). On the expression of joint moments during gait. Gait and Posture, 25, 440-452.

Schwartz, M., \& Romzumalski, A. (2005). A new method for estimating joint parameters from motion data. Journal of Biomechanics, 38, 107-116.

Van Sint Jan, S., \& Della Croce, U. (2006). Identifying the location of human skeletal landmarks: Why standardized definitions are necessary-a proposal. Clinical Biomechanics, 20, 659-660.

Vaughan, C., Davis, B., \& O'Connor, J. (1999). Dynamics of human gait. Cape Town: Kiboho Publishers. 